a model of stroke. Preventing complex formation protected against stroke-induced injury. Cell 149, 1536-1548 (2012)

\section{ASTRONOMY}

\section{Similar orbits but not densities}

Data from NASA's Kepler spacecraft have revealed two extrasolar planets with similar orbits around the same star but radically different densities - challenging planetary formation theories.

Joshua Carter at the Harvard-Smithsonian Center for Astrophysics in Cambridge, Massachusetts, Eric Agol at the University of Washington in Seattle and their team found the two planets, which have orbital periods of 14 and 16 days. The inner planet has a density consistent with a rocky planet like Earth, whereas the outer one has the density of a gaseous planet, such as Neptune. This is puzzling to theorists who had expected gaseous planets to form much further out, as in the Solar System. When viewed from the Earth-like planet, the Neptunelike planet would be 2.5 times the size of the Moon.

Science http://dx.doi.org/

10.1126/science.1223269 (2012)

\section{PHOTONICS}

\section{Data travel on spiralled light}

The amount of information that can be crammed into a beam of light can be increased by splitting the photon stream into different shaped spirals that act as independent channels.

Jian Wang at the Wuhan National Laboratory for Optoelectronics in China, Alan Willner at the University of Southern California, Los Angeles, and their team used the technique to send terabits of information per second through free space. The technique could be combined with more conventional methods of high-density data transfer - such as splitting a beam into two different polarizations or multiple wavelengths - to achieve massive data-flow.

The method could prove particularly well-suited to cable-free information transfer over short distances, or to communication in space. Nature Photon. http://dx.doi. org/10.1038/nphoton.2012.138 (2012)

\section{MOLECULAR BIOLOGY}

\section{Protein crawls on chromosome}

Proteins that control gene expression by attaching to specific DNA sequences amble along chromosomes until they hit their targets, according to a single-molecule imaging study.

In the 1970s, researchers theorized that one such protein, or transcription factor, called the lac repressor (LacI) would act in this way. Johan Elf and his team at Uppsala University in Sweden provide the first evidence of this behaviour in vivo. In Escherichia coli cells, the team found that LacI took 3-5 minutes to bind to the correct DNA site - the speed predicted by theoretical models of 'chromosome crawling. When other proteins are bound to the chromosome, they act like roadblocks and slow the search. Moreover, the transcription factor typically slides over its target several times before binding to it. Science 336, 1595-1598 (2012)

\section{PLANETARY SCIENCE}

\section{Carbon dioxide snow on Mars}

Clouds of snow - frozen carbon dioxide crystals - at the Martian poles are made up of particles just 8-44 micrometres in diameter.

The Martian atmosphere consists mainly of carbon dioxide, which condenses at the poles during the winter. Renyu $\mathrm{Hu}$ and his colleagues at the Massachusetts Institute of Technology in Cambridge analysed temperature, pressure

COMMUNITY CHOICE

The most viewed papers in science

\title{
Biodiverse and language-diverse
}

\section{HIGHLY READ \\ on www.pnas.org in May} contain about $70 \%$ of the world's languages.

Biologists and linguists have previously noted that areas with high species diversity also tend to show high language diversity. The data set, published in 2010, has enabled Larry Gorenflo at Pennsylvania State University in University Park and his colleagues to perform a more detailed analysis. Regions that are particularly rich linguistically include parts of west Africa and southeast Asia and some of the Indonesian islands, which have more than 250 native languages each. These languages are often unique to their regions and many are at risk of extinction.

The authors suggest a range of possible reasons for this link. For one, the spread of a few dominant European languages tended to occur in more temperate areas, and not in the biodiverse tropics.

Proc. Natl Acad. Sci. USA 109, 8032-8037 (2012)

and topography data gathered over more than 9 years by the Mars Global Surveyor and Mars Reconnaissance Orbiter. The researchers found that snow accumulation is greater at the south pole than at the north and that the clouds spread halfway towards the equator before retreating in the spring. J. Geophys. Res. http://dx.doi.org /10.1029/2012JE004087 (2012)

\section{CLIMATE CHANGE}

\section{Cyclones on the move}

Tropical cyclones in the North Indian Ocean will tend to occur later in the year, during the peak monsoon season, as the climate warms.

Hiroyuki Murakami, now at the University of Hawaii at Manoa in Honolulu, and his colleagues used several versions of an atmospheric circulation model to project changes in cyclone activity in the North Indian Ocean in 2075-99 relative to 19792003. The researchers found that, in a simulated warmer future, fewer cyclones formed in the pre-monsoon season

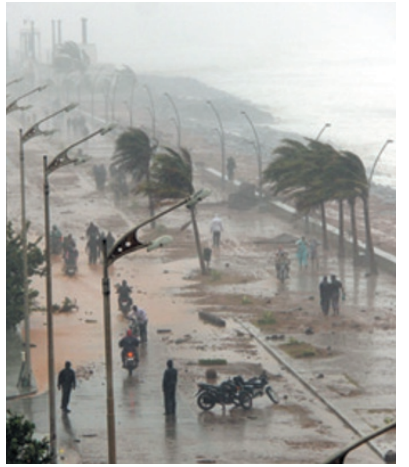

in spring, whereas a greater number of storms occurred in the peak-monsoon season in summer. Overall cyclone frequency in the region did not change significantly, however.

Increased sea surface temperature and a reduction in vertical wind shear - which pulls cyclones apart - could explain the projected increase in storm frequency during the summer monsoon season. Clim. Dyn. http://dx.doi.org/ 10.1007/s00382-012-1407-z (2012)

\section{$\rightarrow$ NATURE.COM}

For the latest research published by Naturevisit:

www.nature.com/latestresearch 